sympathomimetic and sympatholytic agents and drugs acting on sensory systems achieve a more even balance between the raw data of structure-action correlations and theoretical interpretation.

Most of the people working on these various areas of chemical pharmacology will be familiar with the general patterns of chemical structure variation that have been explored. What is most useful in this book is that the various theories which have been proposed are all collected together and extensively discussed. This survey shows that there is no shortage of ideas about the way in which chemical structure influences biological activity, but also elearly reveals the basic problem of chemical pharmacology - that it is extremely difficult to devise critical experiments to support or refute these ideas. Many intriguing problems are discussed and this collection of articles should prove very useful to anyone interested in this difficult problem of the relationship between chemical structure and physiological effect.

This volume is handsomely produced and printed; it is prefaced by an embarrassing piece of American mortuary prose that would have been better omitted; it is also very expensive.

F. W. GIIL

\section{ANTIBIOTICS GIVEN TREATMENT}

\section{Antibiotics}

Origin, Nature and Properties. By Tadeusz Korzybski, Zuzanna Kowszyk-Gindifer and Wlodzimierz Kurylowicz. Translated by Edwin Paryski. Vol. 1: Pp. xix +1-1144. Vol. 2: Pp. xviii +1145-1651. (Oxford, London and New York: Pergamon Press, Ltd.; Warszawa: PW'NPolish Scientific Publishers, 1967.) 294s. net per set of two volumes.

With the explosive preliferation of published data in the field of antibiotics during the past decade, the need for an authoritative English lar guege reference work, embracing combined knowledge of the many aspects of the biology and chemistry of these diverse compounds, has been keenly felt. These two volumes represent a most ambitious attempt to fill this gap, with the very large number of individual antibiotics listed providing its own tribute to the diligent application of the authors to such a herculean task.

The presentation, in view of the virtual impossibility of achieving an ideal classification of the antibiotics, is excellent, with the work being divided into soven main sections: antibioties obtained from $(a)$ the order Eubacteriales, (b) the order Actinomycotales, (c) Fungi imperfecti, (d) Basidiomycetes and Ascomycetes, (e) lichens and algae, $(f)$ higher plants, $(g)$ animal sources, of which the first two sections constitute the first volume and the others constitute the second volume. Further subdivisions are then made according to genus and on the basis of chemical structure, antimicrobial spectra and so on, as best suited. The presentation of many of the data in clear tabular form is a great advantage to the reader. While the inclusion of compounds derived from higher plants and animals as antibiotics is open to debate, it again underlines the wide coverage of the work.

The biological topies, which include accounts of the origin, culture conditions, isolation techniques, antimicrobial spectra, and elinical implications of the various antibiotics, are magnificently handled, as are considerations of stability, physical properties and so on. The copious cross-referencing within the various sub-sections, each of which has its own biblicgraphy, is a most commendable feature. In view of this, it is all the more unfortunate that on the chemical side the work falls short of what might have been achieved.

The authors have largely ignored biogenetic aspects, as evidenced by the controversial statement in the introduction that the biogenesis of the antibiotics remains unclarified, and by the brief dismissal of the biogenesis of various specific compourds such as stipitatic acid and patulin in terms of statements to the effect that this has been studied. The failure to indicate the results of therse biogenetic studies is most sumprising, especially in view of the intense current interest in the topic as evidenced by the many published pspers ard by the appearance of several books devoted to this aspect alone.

An obvious attenipt to update the original Polish version of the work has been made. This has led to some unfortunate situations, such as the clumsy insertion of the correct formulae for verrucarol and its close relatives with the retention of erroneous structures for the derived verrucarins and roridins, and the insertion of the correct formulae of the polymyxins with the retention of an erroneous table which complies noither with earlier spurious assignments of structure nor with the correct structural assignments of polymyxins $B_{1}, B_{2}$ and $E_{1}$. Even so, there are very few post-1964 references to original literature, amerdments to the section on viomycin (where no evidence is presented in support of the revised structure) beirg a notable exception.

In some instances the chemical data are unreliable, indicating that, although the literature survey would appear to extend through 1964 , its breadth in this area is suspect. Specific examples are the incomplete or ineorrect structures given for iridomyrmecin (which, ineidentally, is misspelt), "polyporenic acid", gecdin, erdin, solanocapsine, pleuromutilin, pristimerin and grifolin-to mention random exaryples-where the correct structures were all in the published literature by 1963 , in some cases in the $1950 \mathrm{~s}$. The time interval occasioned by the translation and publication of the English version, of course, precludes the correct portrayal of the structures of important compounds such as fusidic acid, viridin, marasmic acid and hirsutic acid, and also precludes consideration of the stereochemistry and conformational aspects of the streptomycin molecule, as well as discussion of a number of more recently isolated antibiotics.

Portrayal of absolute stereochemistry by means of stereoformulae appears to have been done in a most arbitrary manner, and for important groups such as the penicillins, cephalosporins and tetracyclines there is no stereo portrayal at all.

Nevertheless, despite the shortcomings of these volumes on the chemical side, and in one or two other areas-as with the bacitracins, where more than the three bacitracins listed are known-workers in the many faceted area of antibiotic research will find it hard to resist their acquisition, for they provide a rapid and convenient source of comprehensive information and references to the original literature up to about throe years ago. Purchase of both volumes is mandatory because the comprehensive author and subject indices for the two are in the second volume.

M. Martin-Smith.

\section{MURINE PATHOLOGY}

Pathology of Laboratory Rats and Mice

Edited by Ernest Cotchin and Francis J. C. Roe. Pp. xxiii +848 . (Oxford and Edinburgh: Blackwell Scientific Publications, 1967.) 155s.

THE Nuffield Foundation has recently become interested in the possible long term effects that food additives may have on human health. The screening of such substances to detect possible toxicity often involves tests on laboratory animals, and so the Foundation held a conference in 1966 devoted entirely to the pathology of laboratory rats and mice.

Two of the participants, E. Cotchin and F. J. C. Roe, have edited the twenty.four papers that were presented by recognized authorities and have produced a most comprehensive and welcome monograph. 